\title{
Effects of hand grip strength on shoulder muscle activity in breast cancer patients
}

\author{
Tae-Won Yun ${ }^{a}$, Byoung-Hee Lee ${ }^{b}$ \\ ${ }^{a}$ Department of Rehabilitation, Suwan Rehabilitation Hospital of Suwan Medical Center, Gwangju, Republic of Korea \\ ${ }^{b}$ Department of Physical Therapy, College of Health and Welfare, Sahmyook University, Seoul, Republic of Korea
}

\begin{abstract}
Objective: The purpose of this study was to investigate effects of hand grip strength on the muscle activation of shoulder joint in breast cancer patients.

Design: Cross-sectional study.

Methods: Ten breast cancer patients who agreed to active participation were included. These patients were operated with either conservative surgery or segmental mastectomy, and then were treated with radiation therapy and chemotherapy. The activity of the upper trapezius, lower trapezius, supraspinatus and serratus anterior muscle were measured using surface electrodes during 4 hand gripping tasks (lowered their arms in standing position, $0 \%, 30 \%$, and $50 \%$ of maximum voluntary contraction) in the scapular abduction plane.
\end{abstract}

Results: The results were analyzed using a one-way repeated measures ANOVA. There was a significant difference in the lower trapezius and supraspinatus muscles according to grip strength, lower trapezius and supraspinatus muscles showed significantly difference according to grip strength $(p<0.05)$. The result of the muscle activation according to hand strength $(0 \%, 30 \%$, and $50 \%)$ it showed a significant difference between the upper trapezius and supraspinatus in $0 \%$ grip strength $(p<0.05)$. In addition, it did not show a significant difference between muscles in 30\%, 50\% hand strength.

Conclusions: This study showed an increase in shoulder muscle activation with increasing hand grip strength with the upper trapezius muscle being more activated than other muscles in $0 \%$ grip strength. The finding of this study suggests usefulness for development of preventative measures and rehabilitation strategies for increasing shoulder motor function in patients with breast cancer.

Key Words: Breast cancer, Hand grip strength, Muscle activation, Shoulder motor function

\section{Introduction}

Breast cancer is a common type of cancer in Korean women and has the second highest incidence following thyroid cancer [1]. The number of breast cancer patients in 2011 was 16,967 , four times higher than the number of 15 years before. The incidence of the cancer was 52.1 cases per 100,000 females, an incidence that is higher than those of other Asian nations. Although the domestic mortality has been on the increase due to surge of breast cancer, increase in early diagnosis by active medical examination and application of standardized treatments to patients have caused in- crease in survival rate from the cancer.

Surgical methods for breast cancer can be divided into total mastectomy and breast conserving surgery. Cases of breast conserving surgery (partial mastectomy) have consistently increased from $27.9 \%$ in 2009 to $67.2 \%$ in 2012 , indicating that more than half of patients could conserve their breasts even after they were diagnosed as breast cancer. As for treatment of breast cancer, most patients recently undergo surgery and then radiation therapy, anti-cancer chemotherapy, anti-hormone therapy, and targeted therapy as adjuvant ones for preventing recurrence.

However, surgeries of breast cancer induce sore arm, dif-

Received: 27 April, 2016 Revised: 13 June, 2016 Accepted: June 13, 2016

Corresponding author: Byoung-Hee Lee

Department of Physical Therapy, The Graduate School, Sahmyook University, 815 Hwarang-ro, Nowon-gu, Seoul 01795, Republic of Korea Tel: 82-2-3399-1634 Fax: 82-2-3399-1639 E-mail: 3679@syu.ac.kr

(c) This is an Open-Access article distributed under the terms of the Creative Commons Attribution Non-Commercial License (http://creativecommons.org/licens es/by-nc/4.0) which permits unrestricted non-commercial use, distribution, and reproduction in any medium, provided the original work is properly cited.

Copyright $@ 2016$ Korean Academy of Physical Therapy Rehabilitation Science 
ficulty in sleeping, skin irritation, fatigue, malfunction in the shoulder joints [2], limitation in shoulder joint working range, weakening of hand muscles, pain, discomfort, and lymphedema [3], while radiation therapy causes more decline in functions of the affected upper limb [4]. These problems have negative effects on quality of life related to health as well as daily life of the patients [5]. When 141 breast cancer patients were assessed of their joint functions 18 months after surgeries and radiation therapy [6], 50\% of them showed reduction in the shoulder joint functions when compared to those before the surgeries. A research on 114 breast cancer patients who had underwent surgeries two years (on average) before reported that the working ranges of abduction, external rotation, and internal rotation were sufficiently reduced [7].

The scapulae provide a role of a stabilizer as the fulcrum of movements of the shoulder joints and upper limbs, serving as a dynamic stabilizer [8]. However, cases of pain or arthropathology can induce imbalance of the shoulder joint muscles and damage to scapular arrangement [9]. A research on breast cancer patients reported that the affected scapula after mastectomy was rotated more upwardly than the non-affected one, a rotation that might induce shoulder disorder [10]. In addition, there were significant differences in the scapular location and working range of the joints in resting between the arm with and the arm without lymphedema after breast cancer surgery [11]. Such reports may indicate the necessity of interventions to recover the scapular location for recovery of upper limb function.

The scapulae can serve as a key indicator in assessing upper limb function after breast cancer surgery. During re-

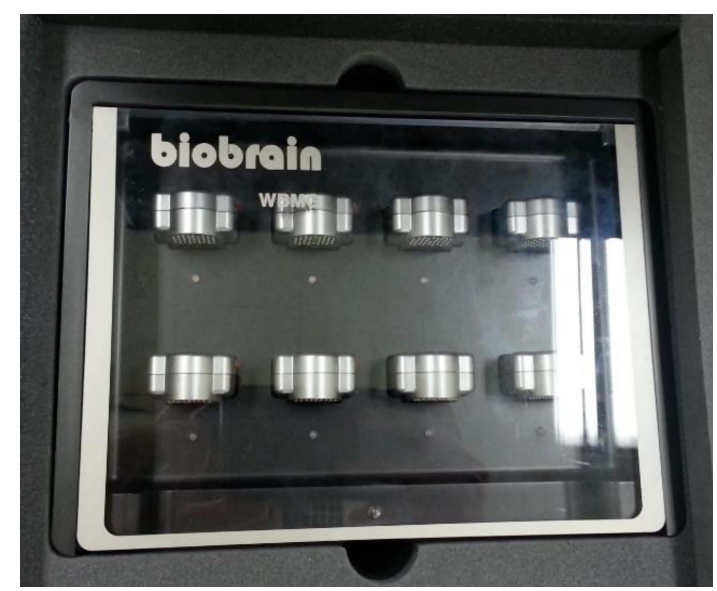

Figure 1. Surface electromyogram system to be used experiment. WBMG8; BioBrain Inc., Daejeon, Korea. habilitation, those who have connective tissue impeding normal scapular movements, differences in muscular length, imbalance of couple of force, or abnormal recruitment patterns of muscle groups need to recover the normal scapular location [12]. We in this study investigated changes in activities of the muscles around the scapulae of breast cancer patients based on grip strength of the affected arm and hand, attempting to suggest basic data for rehabilitation of the patients.

\section{Methods}

\section{Subjects}

The subjects of this study were ten female patients diagnosed with breast cancer who were at least 20 years old and at Stage 1 or Stage 2. Those who had medical history of pathologic illness or surgery on the shoulders, illness on the cervical vertebral portion, systematic illness, or chronic disorder such as uncontrolled high blood pressure or heart diseases were excluded from this study. All the subjects understood the purposes and methods of this study by sufficient explanation before the experiment, which was performed on those who voluntarily showed their consent to participation.

\section{Measurement tools}

\section{Surface electromyogram}

In order to identify changes in activities of muscles affecting stabilization of the scapulae of the subjects, we measured the signals of surface electromyogram (EMG) using the WBMG8 (BioBrain Inc., Daejeon, Korea) as a system of zero-wireless EMG (Figure 1). The measured EMG analogue signals were discrete-digitalized by $1,000 \mathrm{~Hz}$ of sampling frequency for enabling producing indexes in a computing system. The converted digital signals were wirelessly sent to a personal computer, sorted by BioScan (BioBrain Inc.) as this program specifically analyzes EMG, and then was used to produce RMS indexes which reflect muscular activity.

\section{Location of EMG electrodes and normalization}

We rubbed the skin of the subjects with alcohol swabs for cleansing and minimizing skin resistance before the electrodes were attached to the upper trapezius, the lower trapezius, the supraspinous muscle, and the serratus anterior muscle (Figure 2). For the upper trapezius, the electrode was attached slightly medial from the location from the center between the spinous process of the transitional vertebra and the 


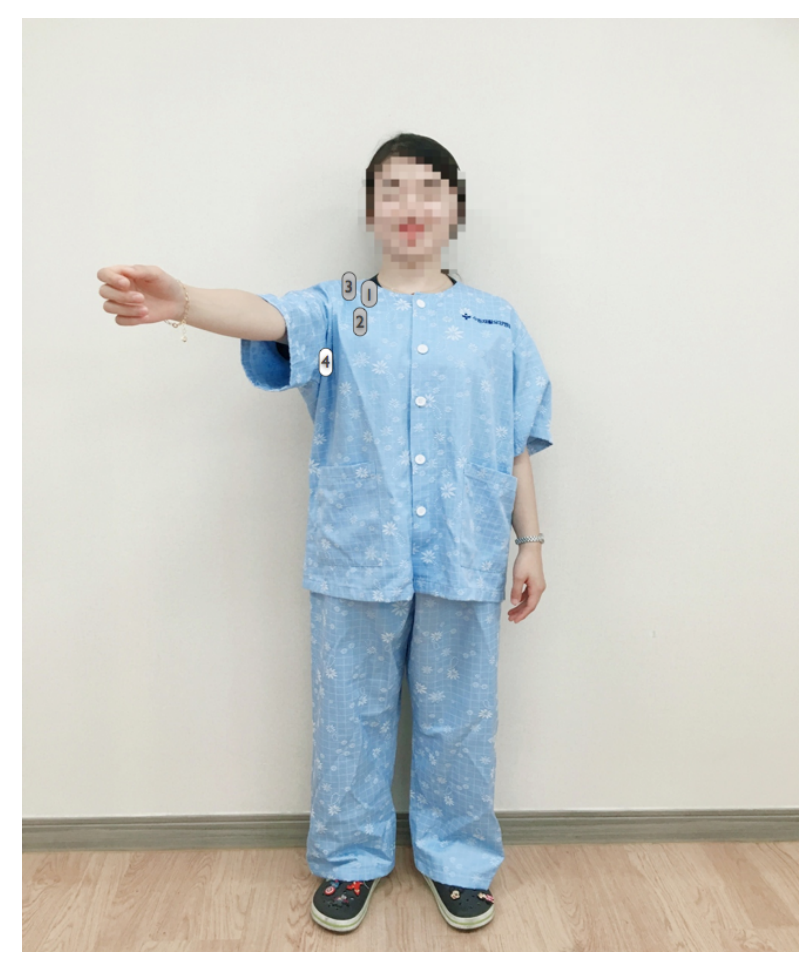

Figure 2. Location of surface EMG electrode. 1: upper trapezius (back), 2: lower trapezius (back), 3: supraspinatus (back), 4: serratus anterior (front).

posterior end of the acromion along the trapezius belly. For the lower trapezius, the electrode was attached to the lateral upper location along the line between spinous process of the eighth thoracic vertebrae and the intersection of the medial border of the scapulae and the spine of scapula. For the supraspinous muscle, the electrode was attached to the supraspinous fossa of $2 \mathrm{~cm}$ above the center of the spine of scapula. For the serratus anterior muscle, the electrode was attached to the center between the lateral margin of the scapulae and the end of the muscle located in the anterolateral thorax [13].

For collecting the RVC, the subjects stood comfortably, facing forward. They stood still and lowered their arms for five seconds, flexed their arms at an angle of 90 degrees of the shoulder joints from the scapular plane for five seconds, and maintained $30 \%$ and $50 \%$ of the maximal grip strength for five seconds. Then, the three-second signals except for pre- and post- one second were used [14]. For normalization of the EMG values, we used \%reference voluntary contraction $(\% \mathrm{RVC})[15]$.

\section{Hand grip digital dynamometer}

We used an electronic dynamometer (Baseline, USA) for
Table 1. Comparison of the general characteristics of the subjects

\begin{tabular}{lcc}
\hline \multicolumn{1}{c}{ Characteristic } & Subjects & $p$ \\
\hline Age (y) & $52.8(5.53)$ & 0.768 \\
Height $(\mathrm{cm})$ & $157.6(4.14)$ & 0.824 \\
Weight $(\mathrm{kg})$ & $59(2.71)$ & 0.844 \\
Prevalence (mo) & $4.9(2.33)$ & 0.887 \\
Stage $1 / 2(\mathrm{n})$ & $6 / 4$ & \\
Location (right/left) & $6 / 4$ & \\
Operation type (conserving & $7 / 3$ & \\
$\quad$ surgery/segmental mastectomy) & & \\
Visual analog scale (score) & $4.3(1.34)$ & 0.466
\end{tabular}

Values are presented as mean (SD).

measuring the hand grip strength. Showing result values by the kilogram based on age and sex, this lightweight electronic spring dynamometer can measure up to $90 \mathrm{~kg}$. The dynamometer used begins measurement when the start button is pressed, and the maximum grip strength during the measurement is automatically calculated and shown on the screen in kilograms.

\section{Analysis}

The collected data were statistically processed using IBM SPSS Statistics ver. 19.0 for Windows (IBM Co., Armonk, NY, USA). Shapiro-Wilk analysis was performed in order to verify normality of the data.

We performed repeated one-way ANOVA in order to compare changes in the muscle activity based on grip strength of the upper trapezius, the lower trapezius, the supraspinatus, and the serratus anterior muscle, while performing one-way ANOVA in order to compare muscular activity values based on grip strength of the upper trapezius, the lower trapezius, the supraspinatus, and the serratus anterior muscle. Bonferoni method was used as a post hoc for each analysis. For all the statistical significance level, $\alpha=0.05$.

\section{Results}

Table 1 presents the mean and standard deviation values of the general characteristics of the subjects. Differences in age, height, weight, and prevalence were observed in the group.

There were no significant differences in grip strength based on the results of the repeated one-way ANOVA. Table 2 shows the comparisons of the muscle activity based on the grip strength intensity of the upper trapezius muscle, the 
Table 2. Comparison of shoulder muscles activity according to hand grip strength on scapular plane $(\mathrm{N}=10)$

\begin{tabular}{|c|c|c|c|c|}
\hline \multirow{2}{*}{ Muscle } & \multicolumn{3}{|c|}{ Hand grip strength } & \multirow{2}{*}{$p$} \\
\hline & $0 \%$ & $30 \%$ & $50 \%$ & \\
\hline Upper trapezius (kg) & $210.70(92.44)^{\mathrm{a}}$ & $220.15(89.74)$ & $337.52(191.56)$ & 0.143 \\
\hline Lower trapezius (kg) & $159.67(49.53)$ & $229.82(82.37)$ & $256.36(121.08)$ & $\begin{array}{c}0.033 \\
\mathrm{~A} / \mathrm{B}, \mathrm{C}\end{array}$ \\
\hline Supraspinatus (kg) & $111.03(32.01)$ & $211.68(101.84)$ & $350.42(251.74)$ & $\begin{array}{c}0.019 \\
\mathrm{~A} / \mathrm{B}, \mathrm{C}\end{array}$ \\
\hline Serratus anterior $(\mathrm{kg})$ & $162.73(77.19)$ & $178.67(83.49)$ & $261.89(169.11)$ & 0.144 \\
\hline$p$ & 0.021 & 0.611 & 0.571 & \\
\hline
\end{tabular}

Values are presented as mean (SD).

A: $0 \%$ of grip strength, B: $30 \%$ of grip strength, C: $50 \%$ of grip strength.

${ }^{\mathrm{a}}$ Significant difference compared with supraspinatous $(p<0.05)$.

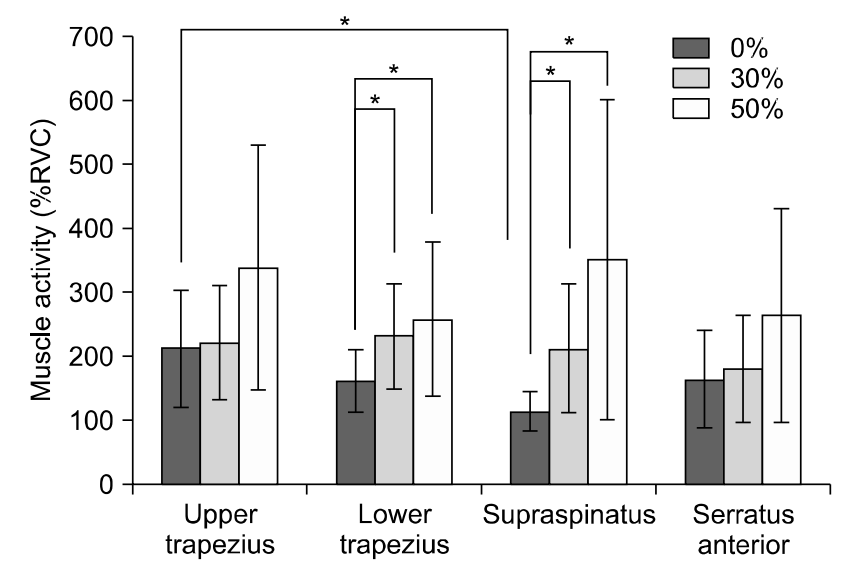

Figure 3. Comparison of shoulder muscles activity according to hand grip strength $\left({ }^{*} p<0.05\right)$.

lower trapezius muscle, the supraspinous muscle, and the serratus anterior muscle (Figure 3). There were no statistically significant differences in the lower trapezius and the supraspinatus, but the muscular activity was high in the order of $0 \%, 30 \%$, and $50 \%$. The muscular activity of the upper trapezius and the serratus anterior muscle was high in the order of $0 \%, 30 \%$, and $50 \%$, and significant differences were observed among $0 \%, 30 \%$, and 50\%.

When the muscle activity of the upper trapezius, the lower trapezius, the supraspinatus, and the serratus anterior muscle was compared on the basis of grip strength intensity, significant differences were found between the upper trapezius and the supraspinatus at $0 \%$ while no significant differences were found among the muscles at $30 \%$ and $50 \%$.

\section{Discussion}

In this study we compared the muscle activity of the upper trapezius, the lower trapezius, the supraspinatus, and the serratus anterior muscle when 10 breast cancer patients performed grasping at $0 \%, 30 \%$, and $50 \%$ of their maximal grip strength. The results showed that the muscle activity was higher in the order of $0 \%, 30 \%$, and $50 \%$ in all the four muscles.

In this study, there were significant differences between the supraspinatus and the upper trapezius of the breast cancer patients at resting $(0 \%)$, and the muscular activity was most outstanding in the upper trapezius. According to a research in which activity of the upper trapezius was investigated between a group with and a group without shoulder joint disorder, the activity of the upper trapezius was considerably higher in the shoulder joint disorder group [16]. Another research reported that the activity of the upper trapezius increased more than that of other muscles. Such results may indicate that the activity of upper trapezius increases in patients with lesions in their shoulder joints. In addition, the activity of the upper trapezius may be related to clavicular elevation or scapular elevation [17-19]. The activity of the upper trapezius shown in patients with such lesions is regarded as a general compensation in performing upper limb functions, and it can be said that the compensation of the upper trapezius is used in cases of breast cancer patients.

As for the muscular activities of the supraspinatus, the infraspinatus, and the trapezius based on $30 \%$ and $50 \%$ of grip strength, the supraspinatus and the infraspinatus showed positive correlation between grip strength and shoulder elevation, and the muscular activity was higher as the grip strength was increased [20]. A research reported that the rest rotator cuff related muscles as well as the supraspinatus contributed to the movement in abduction [21], and many pre- 
vious researches reported that not a single muscle but a functional unit performed movements in conducting a task [22-24].

In a research of comparing muscle activities of the supraspinatus, the infraspinatus, the lower trapezius, the anterior deltoid, and the posterior deltoid based on elevated postures of the arm and grip strength in normal persons, the muscle activities of the shoulder muscles were changed on the basis of grip strength intensity [14]. The researchers suggested that the results might be caused by increase in actions of stability muscles such as the infraspinatus muscle that enable the humeral head to cling to the glenoid fossa when the grip strength induces co-contraction of the proximal and the distal parts of the arm. In this study, increase in the grip strength enhanced the activities of the muscles around the shoulders, indicating that the results consistent with those of the previous researches could be induced via the similar mechanism. A research on associations of grip strength with the muscle group stabilizing the shoulder joints in 18 normal people reported that the grip strength affected the muscle group [25]. According to the researchers, an effective movement could be performed by conducting a movement by the distal joints when the proximal joints firmly stabilized the joints by recruiting the surrounding muscles.

A research reported that the correlation between the proximal joint and the distal joint might be caused by the fact that forces can be delivered through the myofascial pathway between the sarcomeres and the endomysium to the proximal upper limbs [26]. Meanwhile, there is a research on animals to identify the mechanism [27], which showed that forces could be delivered between intra- and extra-muscular connective tissues such as the fascia. The research reported that the results reflected there were borders formed between the extra-muscular force transmission within compartment and the inter-muscular force transmission of the connective tissues that were adjacent to the two muscles within the muscle group. Such anatomical connections may emphasize that significance of appropriate assessment of the shoulder joint complex including the scapulothoracic joint in functionally using the hands in daily life.

Assessment of the scapulothoracic joint has been generally ignored in rehabilitation, and there are even researches reporting that the strong scapular muscles serve as a key prerequisite for securing functional movements and proper stability of the upper limbs [28,29]. The scapular muscles play a significant role in controlling the location of the glenoid fossa, and can have influence on production or alignment of forces as well as movements around the shoulder joint complex though their working ranges are relatively small [30,31]. Such results may indicate that muscular imbalance in the scapulothoracic joint related to the weakened rotator cuff muscles have negative influence on performing daily life movements of the breast cancer patients and that the glenohumeral muscles and the scapular muscles should be consistently observed during rehabilitation of the patients.

While previous researches focused on normal people or patients with shoulder disorder, this study investigated changes in the activities of the four muscles based on grip strength in patients with breast cancer after surgery. However, we did not understand differences from comparison of activities of the muscles around the shoulder joints between breast cancer patients as the subjects of this study and patients with other shoulder disorders. Further studies should include various types of subjects in order to consider specific methods of exercise for solving functional problems of breast cancer patients.

Some limitations of this study were related to the reduced range of motion used to assess the scapular protraction/retraction movements and the relatively small sample size. The limited range of motion was determined to minimize possible compensatory movements of the trunk and the utilization of stronger muscular groups to perform the scapular movements. Lastly, further research is needed to investigate the difference from the comparison of muscle activities around the shoulder joints between breast cancer patients and patients with other shoulder disorders

In this study we compared the muscle activities in the shoulder joints of breast cancer patients based on grip strength after surgery and the results showed that the activities of the shoulder joints were higher as the grip strength was more intense. Thus, grip strength may be needed to provide in performing exercises during rehabilitation for improving the upper limb functions of breast cancer patients.

\section{Conflict of Interest}

The authors declared no potential conflicts of interest with respect to the authorship and/or publication of this article.

\section{References}

1. Jung KW, Won YJ, Kong HJ, Oh CM, Cho H, Lee DH, et al. Cancer statistics in Korea: incidence, mortality, survival, and prevalence in 2012. Cancer Res Treat 2015;47:127-41. 
2. Longman AJ, Braden CJ, Mishel MH. Pattern of association over time of side-effects burden, self-help, and self-care in women with breast cancer. Oncol Nurs Forum 1997;24:1555-60.

3. McNeely ML, Campbell K, Ospina M, Rowe BH, Dabbs K, Klassen TP, et al. Exercise interventions for upper-limb dysfunction due to breast cancer treatment. Cochrane Database Syst Rev 2010;(6):CD005211.

4. Ryttov N, Blichert-Toft M, Madsen EL, Weber J. Influence of adjuvant irradiation on shoulder joint function after mastectomy for breast carcinoma. Acta Radiol Oncol 1983;22:29-33.

5. Ashikaga T, Krag DN, Land SR, Julian TB, Anderson SJ, Brown AM, et al. Morbidity results from the NSABP B-32 trial comparing sentinel lymph node dissection versus axillary dissection. $\mathrm{J}$ Surg Oncol 2010;102:111-8.

6. Sugden EM, Rezvani M, Harrison JM, Hughes LK. Shoulder movement after the treatment of early stage breast cancer. Clin Oncol (R Coll Radiol) 1998;10:173-81.

7. Gaskin TA, LoBuglio A, Kelly P, Doss M, Pizitz N. STRETCH: a rehabilitative program for patients with breast cancer. South Med J 1989;82:467-9.

8. Park JY, Lhee SH, Oh JH, Kim HK. Scapular dyskinesis. J Korean Shoulder Elbow Soc 2009;12:271-7.

9. Sahrmann S. Diagnosis and treatment of movement impairment syndromes. St. Louis: Mosby; 2002.

10. Crosbie J, Kilbreath SL, Dylke E, Refshauge KM, Nicholson LL, Beith JM, et al. Effects of mastectomy on shoulder and spinal kinematics during bilateral upper-limb movement. Phys Ther 2010;90:679-92.

11. Ha HJ, Ahn SY, Kwon HY. The relationship between upper limb lymphedema after mastectomy and scapular dyskinesis. Korean J Sport Sci 2013;22:1103-12.

12. Park JS, Jeon HS, Kwon OY. A comparison of the shoulder stabilizer muscle activities during push-up plus between persons with and without winging scapular. Korean Res Soc Phys Ther 2007; 14:44-52.

13. Heinrichs K. Introduction to surface electromyography. J Athl Train 1999;34:69.

14. Jang HJ, Kim JS, Choi JD, Kim SY. The effects of hand grip force on shoulder muscle activity in two arm posture. J Korea Acad-Ind Coop Soc 2012;13:1229-37.

15. Pontillo M, Orishimo KF, Kremenic IJ, McHugh MP, Mullaney MJ, Tyler TF. Shoulder musculature activity and stabilization during upper extremity weight-bearing activities. N Am J Sports Phys Ther 2007;2:90-6.

16. Peat M, Grahame RE. Electromyographic analysis of soft tissue lesions affecting shoulder function. Am J Phys Med 1977; 56:223-40.

17. Lukasiewicz AC, McClure P, Michener L, Pratt N, Sennett B. Comparison of 3-dimensional scapular position and orientation between subjects with and without shoulder impingement. J Orthop Sports Phys Ther 1999;29:574-83; discussion 584-6.

18. McClure PW, Michener LA, Karduna AR. Shoulder function and 3-dimensional scapular kinematics in people with and without shoulder impingement syndrome. Phys Ther 2006;86:1075-90.

19. Burkhart SS, Morgan CD, Kibler WB. The disabled throwing shoulder: spectrum of pathology Part III: The SICK scapula, scapular dyskinesis, the kinetic chain, and rehabilitation. Arthroscopy 2003;19:641-61.

20. Sporrong H, Palmerud G, Herberts P. Hand grip increases shoulder muscle activity, an EMG analysis with static hand contractions in 9 subjects. Acta Orthop Scand 1996;67:485-90.

21. Sharkey NA, Marder RA, Hanson PB. The entire rotator cuff contributes to elevation of the arm. J Orthop Res 1994;12:699-708.

22. Michiels I, Bodem F. The deltoid muscle: an electromyographical analysis of its activity in arm abduction in various body postures. Int Orthop 1992;16:268-71.

23. Jensen $C$. The surface electromyographic (EMG) amplitude as an estimate of upper trapezius muscle activity. Trondheim: University of Trondheim, Department of Zoology; 1995.

24. Kadaba MP, Cole A, Wootten ME, McCann P, Reid M, Mulford $\mathrm{G}$, et al. Intramuscular wire electromyography of the subscapularis. J Orthop Res 1992;10:394-7.

25. Mandalidis D, O'Brien M. Relationship between hand-grip isometric strength and isokinetic moment data of the shoulder stabilisers. J Bodyw Mov Ther 2010;14:19-26.

26. Huijing PA. Muscular force transmission necessitates a multilevel integrative approach to the analysis of function of skeletal muscle. Exerc Sport Sci Rev 2003;31:167-75.

27. Huijing PA, Baan GC. Myofascial force transmission: muscle relative position and length determine agonist and synergist muscle force. J Appl Physiol (1985) 2003;94:1092-107.

28. Cools AM, Geerooms E, Van den Berghe DF, Cambier DC, Witvrouw EE. Isokinetic scapular muscle performance in young elite gymnasts. J Athl Train 2007;42:458-63.

29. Moraes GF, Faria CD, Teixeira-Salmela LF. Scapular muscle recruitment patterns and isokinetic strength ratios of the shoulder rotator muscles in individuals with and without impingement syndrome. J Shoulder Elbow Surg 2008;17(1 Suppl):48S-53S.

30. Cools AM, Witvrouw EE, Danneels LA, Vanderstraeten GG, Cambier DC. Test-retest reproducibility of concentric strength values for shoulder girdle protraction and retraction using the Biodex isokinetic dynamometer. Isokinet Exerc Sci 2002;10: 129-36.

31. Phadke V, Camargo P, Ludewig P. Scapular and rotator cuff muscle activity during arm elevation: a review of normal function and alterations with shoulder impingement. Rev Bras Fisioter 2009;13:1-9. 